\title{
SUBSTITUTE NAMES FOR SOME EXTINCT GENERA OF FOSSIL INSECTS*
}

\author{
By Frank M. Carpenter \\ Museum of Comparative Zoology, Harvard University \\ Cambridge, MA 02138
}

During the preparation of the hexapod volume of the Treatise on Invertebrate Paleontology, now in press $\dagger$, several junior homonyms were noted among the extinct genera. This seems to be an appropriate occasion to propose substitute names for these homonyms. In those instances in which the authors are still living, I have already called their attention to the homonymy, with the suggestion that they propose names of their own choice. In some other cases replacement names are already available, through synonymy, and these are being proposed in the hexapod volume of the Treatise. Most of the homonyms, however, require new names, and since the current editorial policy of the Treatise excludes the proposal of new names of taxa in that work, the present article is intended to meet that need. It consists of proposals of new generic names to replace certain names that are not being taken care of elsewhere. The gender of each new name is the same as that of the original one. The names proposed here are in the orders Palaeodictyoptera, Odonata, Diptera, Hymenoptera, Coleoptera, and Lepidoptera.

\section{Order Palaeodictyoptera}

Family uncertain

Boltonocosta, nomen novum pro ORTHOCOSTA Bolton, 1912, p. 310, non Fritsch, 1879, p. 28. Type-species: Orthocosta splendens Bolton, 1912, original designation, becomes Boltonocosta splendens (Bolton), new combination. The genus is known only from the Upper Carboniferous of England.

Eurydictyella, nomen novum pro EURYDICTYA Guthörl, 1934, p. 49 , non Ulrich, 1889 , p. 301 . Type-species: Eurydictya

\footnotetext{
*This research was supported by National Science Foundation Grant DEB 8205398 , F. M. Carpenter, Principal Investigator.

$\dagger$ Part R, Arthropoda 4, being published jointly by the Geological Society of America and the University of Kansas.
} 
richteri Guthörl, 1934, original designation, becomes Eurydictyella richteri (Guthörl), new combination. The genus is known only from the Upper Carboniferous of Germany.

Order Odonata

Family Aeshnidae

Neoligaeschna, nomen novum pro Oligoaeschna Piton \& Theobald, 1939, p. 6, non Selys, 1889, p. 160. Type-species: Oligoaeschna jungi Piton \& Theobald, 1939, original designation, becomes Neoligaeschna jungi (Piton \& Theobald), new combination. The genus is known only from the Oligocene of France.

\section{ORDER DiPTERA}

\section{Family Architipulidae}

Leptotipuloides, nomen nudum pro LePTotipula Bode, 1953, p. 312, non Alexander, 1917, p. 160. Type species: Leptotipula fastigata Bode 1953, original designation, becomes Leptotipuloides fastigata (Bode), new combination. The genus is known only from the Jurassic of Germany.

Family Bibionidae

Lithosomyia, nomen novum pro Mesomyia Pongracz, 1928, p. 174, non Macquart, 1849, p. 341. Type-species: Bibio brevis Heer, 1849, p. 225, SD Carpenter, herein, becomes Lithosomyia brevis (Heer), new combination. The genus is known by several species from the Miocene of Croatia.

Family Rachiceridae

Trecela, nomen novum pro EleCtra Loew, 1850, p. 38, non Stevens, 1831, p. 278. Type-species: Electra formosa Loew, 1850, original designation, becomes Trecela formosa (Loew), new combination. The genus is known only from the Lower Oligocene of the Baltic amber, The name Trecela is an anagram of Electra and is considered feminine.

\section{ORDER HYMENOPTERA}

Family Eumenidae

Eunortonia, nomen novum pro PSEUdONORTONIA TimonDavid, 1944, p. 41, non Soika, 1936, p. 168. Type-species: Pseudonortonia sepulta Timon-David, 1944, original designation, becomes Eunortonia sepulta (Timon-David), new combination. The genus is known only from the Oligocene of France. 
Family Chrysididae

Protochrysidis, nomen novum pro ProTOCHRYsis Bischoff, 1917, p. 139, non Pascher, 1911, p. 191. Type-species: Protochrysis succinalis Bischoff, 1917, original designation, becomes Protochrysidis succinalis (Bischoff), new combination. The genus is known only from the Lower Oligocene of the Baltic amber.

\section{ORDER COLEOPTERA}

Family Curculionidae

Oligocryptus, nomen novum pro EUCRYPTUS Scudder, 1893, p. 63, non Haldeman, 1842, p. 191. Type-species: Eucryptus sectus Scudder, 1893, original designation, becomes Oligocryptus sectus (Scudder), new combination. The genus is known only from the Oligocene of Colorado.

Sitonitellus, nomen novum pro SITONITES Haupt, 1956, p. 80, non Heer, 1865, p. 90. Type-species: Sitonites egregius Haupt, 1956, original designation, becomes Sitonitellus egregius (Haupt), new combination. The genus is known only from the Eocene of Germany.

Family unknown

Aptilotitus, nomen novum pro APTILotus Bode, 1953, p. 237, non Mik, 1898, p. 206. Type-species: Aptilotus capitecarens Bode, 1953, original designation, becomes Aptilotitus capitecarens (Bode), new combination. The genus is known only from the Jurassic of Germany.

Auchenophorites, nomen novum pro Auchenophorus Bode, 1953, p. 229, non Turner, 1907, p. 270. Type-species: Auchenophorus sculpturatus Bode, 1953, original designation, becomes Auchenophorites sculpturatus (Bode), new combination. The genus is known only from the Jurassic of Germany.

Barocephalus, nomen novum pro BARYCEPHALUs Bode, 1953, p. 228, non Guenther, 1860, p. 149. Type-species: Barycephalus nudatus Bode, 1953, original designation, becomes Barocephalus nudatus (Bode). The genus is known only from the Jurassic of Germany.

Clinomerus, nomen novum pro CATOMERUs Handlirsch, 1939, p. 67, non Pilsbry, 1916, p. 395 . Type-species: Catomerus laticollis Handlirsch, 1939, original designation, becomes 
Clinomerus laticollis (Handlirsch), new combination. The genus is known only from the Jurassic of Germany.

Critoderma, nomen novum pro CyCloderma Heer, 1865, p. 89, non Peters, 1854, p. 216. Type-species: Cycloderma deplanatum Heer, 1865, original designation, becomes Critoderma deplanatum (Heer), new combination. The genus is known only from the Jurassic of Switzerland.

Critotrachelus, nomen novum pro Cyclotrachelus Bode, 1953, p. 222, non De Chaudoir, 1838, p. 27. Type-species: Cyclotrachelus exsecatus Bode, 1953, original designation, becomes Critotrachelus exsecatus (Bode), new combination. The genus is known only from the Jurassic of Germany.

Dicyphelus, nomen novum pro DicYPHUS Bode, 1953, p. 234, non Fieber, 1858, p. 327. Type-species: Dicyphus concameratus Bode, 1953, original designation, becomes Dicyphelus concameratus (Bode), new combination. The genus is known only from the Jurassic of Germany.

Eurynotellus, nomen novum pro EURYNOTUS Bode, 1953, p. 207, non Kirby, 1819, p. 418. Type-species: Eurynotus brevicollis Bode, 1953, original designation, becomes Eurynotellus brevicollis (Bode), new combination. The genus is known only from the Jurassic of Germany.

Heeriaopsis, nomen novum pro HeERIELla Handlirsch, 1906, p. 401, non Meunier, 1904, p. 86. Type-species: Elytridium laevigatum Heer, 1878, p. 196, original designation, becomes Heeriaopsis laevigatum (Handlirsch), new combination. The genus is known only from the Triassic of Sweden.

Mesolobites, nomen novum pro LoBites Dunstan, 1923, p. 53, non Mojsisovics, 1873, p. 155. Type species: Lobites tuberculatus Dunstan, 1923, original designation, becomes Mesolobites tuberculatus (Dunstan), new combination. The genus is known only from the Triassic of Australia.

Mesoncus, nomen novum pro Loxoncus Bode, 1953, p. 218, non Schmidt-Goebel, 1846, p. 4. Type-species: Loxoncus procerus Bode, 1953, original designation, becomes Mesoncus procerus (Bode), new combination. The genus is known only from the Jurassic of Germany.

Oligovarus, nomen novum pro VARUS Schlechtendahl, 1894, p. 209 non Stål, 1865, p. 141. Type-species: Varus ignotus Schlechtendahl, 1894, original designation, becomes Oligo- 
varus ignotus (Schlechtendahl), new combination. The genus is known only from the Oligocene of Germany.

Ooidellus, nomen novum pro OoIDEs Bode, 1953, p. 234, non Agassiz, 1846 (Index, p. 260). Type-species: Ooides denudatus Bode, 1953, original designation, becomes Ooidellus denudatus (Bode), new combination. The genus is known only from the Jurassic of Germany.

Parnosoma, nomen novum pro Pedinosoma Bode, 1953, p. 235, non Reibisch, 1893, p. 252. Type-species: Pedinosoma detectum Bode, 1953, original designation, becomes Parnosoma dectectum (Bode), new combination. The genus is known only from the Jurassic of Germany.

Peridosoma, nomen novum pro Perosoma Bode, 1953, p. 217, non Bronn, 1862, p. 135. Type-species: Perosoma praecisum Bode, 1953, original designation, becomes Peridosoma praecism (Bode), new combination. The genus is known only from the Jurassic of Germany.

\section{ORDER LEPIDOPTERA}

\section{Family Nymphalidae}

Jupitellia, nomen novum pro JUPITERIA Scudder, 1889, p. 488, non Bellardi, 1875, p. 20. Type-species: Jupiteria charon Scudder, 1889, original designation, becomes Jupitellia charon (Scudder), new combination. The genus is known only from the Oligocene of Colorado, U.S.A.

\section{Literature Cited}

Agassiz, Louis

1846. Nomenclatoris Zoologici, Index Universalis, $393 \mathrm{pp}$.

Alexander, C. P.

1917. The crane-flies of South Africa in the South African Museum: Annals of the South African Museum, 17: 139-184.

Bellardi, L.

1875. Monografia delle nuclidi trovate finora nei terreni terziari del Piemonte e della Liguria: Il R. Liceo Gioberti nell' Anno scolastico. 32 pp.

BischOFF, HANS

1917. Bernsteinhymenopteren: Beiträge zur Naturkunde Preussens Physikalisch-Oekonomischen Gesellachft Königsberg, 56: 139-144.

Bode, ARNOLD

1953. Die Insektenfauna des Ostnieders achsischen Oberen Lias: Paleontographica (A), 103: 1-375. 


\section{BOLTON, HeRberT}

1912. Insect-remains from the Midland and South-Eastern Coal Measures: Quarterly Journal of the Geological Society of London, 68: 310-323.

BRONN, H. G.

1862. Klassen und Ordnungen Weichtiere (Malacozoa), vol. 3 (1), 135 pp.

Chaudoir, $\mathrm{M}$.

1938. Tableau d'une nouvelle subdivision de genre Feronia Dejean, suivi d'une characteristique de trois nouveaux genres du Carabiques: Bulletin de la Societé Imperiale Naturalistes Moscou, 11(1): 1-32.

Dunstan, B.

1923. Mesozoic insects of Queensland. Part 1. Introduction and Coleoptera: Geological Survey of Queensland Publication, 273: 1-74.

Fieber, F. X.

1858. Criterien zur generischen Theilung der Phytocoriden: Wiener Entomologische Monatschrif, 2: 289-327.

FritsCh, ANTON

1879. Fauna der Haskole und der Kalksteine der Perm-Formation Bohmens, vol. 1, 83 pp.

GUENTHER, AlberT

1860. Contributions to a knowledge of the reptiles of the Himalaya Mountains: Proceedings of the Zoological Society of London, 28: 148-175.

GUThÖRL, PAUL

1934. Die Arthropoden aus dem Carbon und Perm des Saar-Nahe-PfalzGebietes: Abhandlungen der Königliche Preussichen Geologischen Landesanstalt, 164: 1-219.

HaLdeman, S. S.

1842. Remarks on changes of nomenclature in Natural History: Proceedings of the Academy of Natural Sciences, Philadelphia, 1: 1.

HANDLIRSCH, ANTON

1906. Die fossilen Insekten und die Phylogenie der rezentěn Formen, 640 pp. (Leipzig).

1939. Neue Untersuchungen über die fossilen Insekten, mit Erganzungen und Nachragen palaeogeographische und allgemein biologische Probleme.

Haupt, HermanN II: Annalen Naturhistorisches Museum in Wien, 48: 1-240.

1956. Beitraeg zur Kenntnis der eözanen Arthropodenfauna des Geiseltales: Nova Acta Leopoldina, 18(128): 1-90.

HERR, OSWALD

1849. Die Insektenfauna der Tertiargebilde von Oeningen und Radoboj. 2. Heuschrecken, florfliegen, aderflügen schmitterlinge und fliegen: Neue Denkschriften der Allgemeinen Schweizerischen Gesellschaft für die gesamten naturwissenschaften. $264 \mathrm{pp}$. (Leipzig).

1865. Die Urwelt der Schweiz. 628 pp. (Zurich).

LOEW, H. F.

1850. Ueber den Bernstein und die Bernsteinauna: Programm der Königlichen Realschule zu Meseritz. 48 pp. 
Macquart, JeAN

1850. Facultés interieures des animaux invertebres: Memoires de la Societé des Sciences, Lille, 1849, suppl., 129-400.

MeUnier, Fernand

1904. Monographie des Cecidomyidae, des Sciaridae, des Mycetophilidae, et des Chironomidae de l'ambre de la Baltique: Annales de la Societé Scientifique de Bruxelles, 28: 12-264.

MIK, J.

1898. Altes und Neues über Diptera: Wiener entomologische Zeitung, 17: 196-219.

Miller, S. A.

1889. In Ulrich, et al., North American Geology and Paleontology. 664 pp. (Cincinnati).

Mojsisovics, E. V.

1873. Das Gebirge um Hallstatt; Abt. 1, Die Cephalopoden der Hallstatter Kalke: Abhandlungen der K.K. geologischen Reichsanstalt Wien, 6(1), heft 2, p. 1-356.

PASCHER, ADOLPH

1911. Zwei Braune Flagellaen: berichte der deutschen botanischen Gesellschaft, 29: 517-523.

Peters, Wilhelm

1854. Uebersict der auf seiner Reise nach Mossambique beobachteten Schildkröten: Monatsbericht der Preussischen Akademie der Wissenschaften zu Berlin, 1854, p. 215-216.

Pilsbry, H. A.

1916. The sessile barnacles (Cirripedia) contained in the collections of the U.S. National Museum; including a monograph of the American species: Bulletin of the U.S. National Museum, 93: 1-335.

Piton, L. E. \& N. Theobald

1939. Poissons, crustaces et insectes fossiles de l'Oligocene du Puy-de-Muir (Euvergne).: Memoires de la Societé des Sciences de Nancy, 1939: 1-45.

Pongrácz, A.

1928. Die Fossilen insekten von Ungarn, mit besondered Berucksichtugung der Entwicklung der Europaischen Insecten-fauna: Annales HistoricoNaturales Musei Nationalis Hungarici, 25: 91-194.

REIBISCH, J.

1893. Wissenschaftliche Mitteilungen. 3. Die Phylodociden der Plankton Expedition. PP. 248-255.

SChlentendal, D. H. R. von

1894. Beträge zur Kenntnis fossiler Insekten aus dem Braunkohlengeburge von Rott am Siebengebirge: Abhandkungen der Naturforchenden Gesellschaft zu Halle, 20: 197-228.

SChmidt-Goebel, H. M.

1846. Faunula Coleopterorum Birmanicae, adjectis nonnulis Bengaliae indigenis. PP. 1-94. (Prague). 
SCUDDER, S. H.

1893. Tertiary rhyncophorous Coleoptera of the United States: U.S. Geological Survey Monograph, 21: 1-206.

Selys-LongChamps, E. DE

1889. Odonates de Sumatra comprenant des éspeces recueillies à Pulo Nias par M. le Dr. E. Modigliana: Annali de Museo civico di naturae di Genova, 27: 444-480.

SoIKA, A. G.

1936. Caratteri del gen, Nortonia Sauss. e descrizione due nouve specie: Annali del Museo Civico Storia naturae di Genova, 59: 267-271.

STA L L CARL

1865. Hemiptera Africana, Holmia officina norsted-tiana, vol. 3, pp. 356.

Stevens, J. F.

1931. Illustrations of British Entomology, vol. 3, pp. 1-374.

TURNer, R. E.

1907. New species of Sphegidae from Australia: Annals \& Magazine of Natural History, (7) 19: 268-276.

Timon-David, JEAN

1944. Insectes fossiles de l'Oligocene inferieur des Camoins (Bassin lde Marseille). II: Hymenopteres: Bulletin de la Societé Entomologique de France, 49: 40-45. 

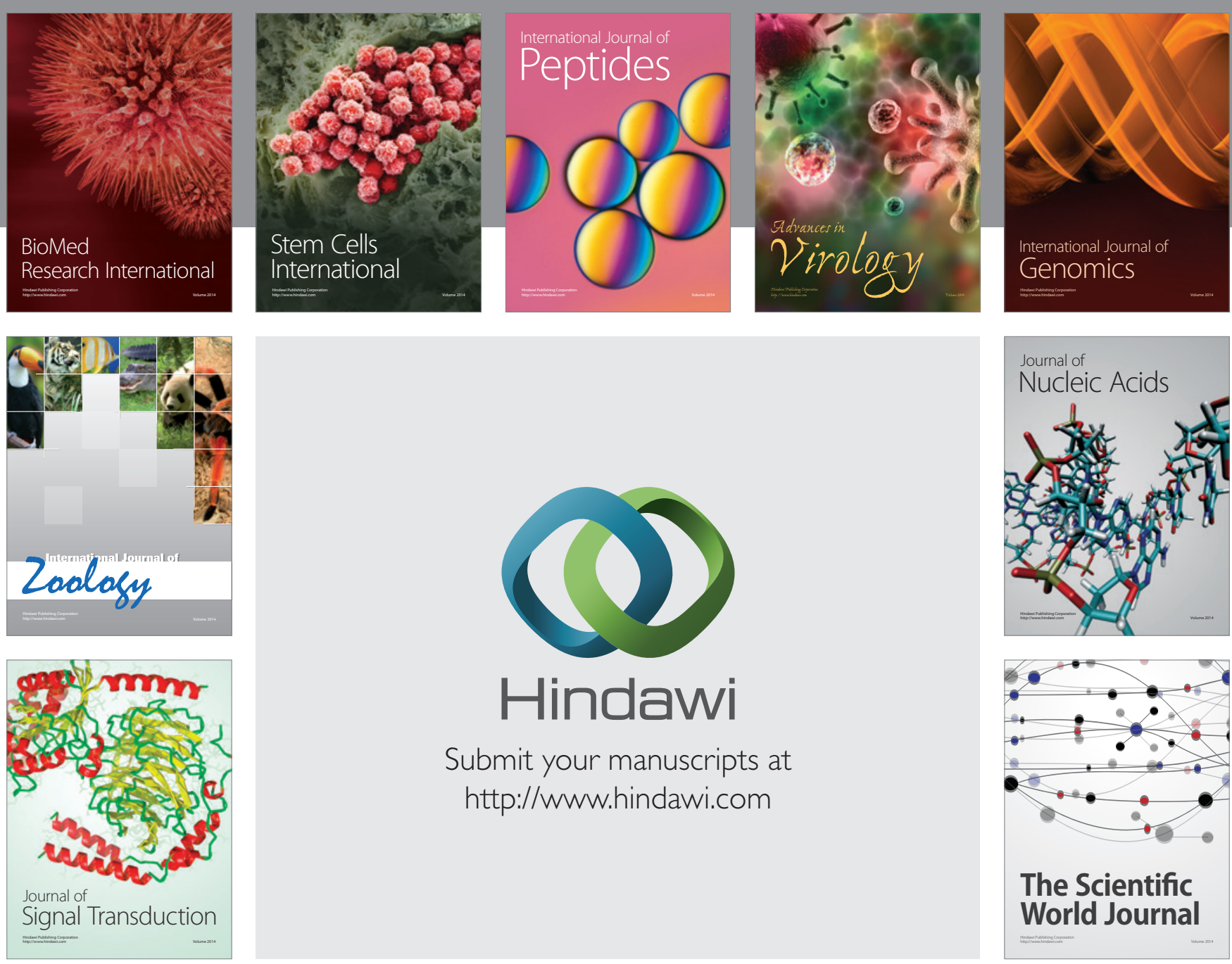

Submit your manuscripts at

http://www.hindawi.com
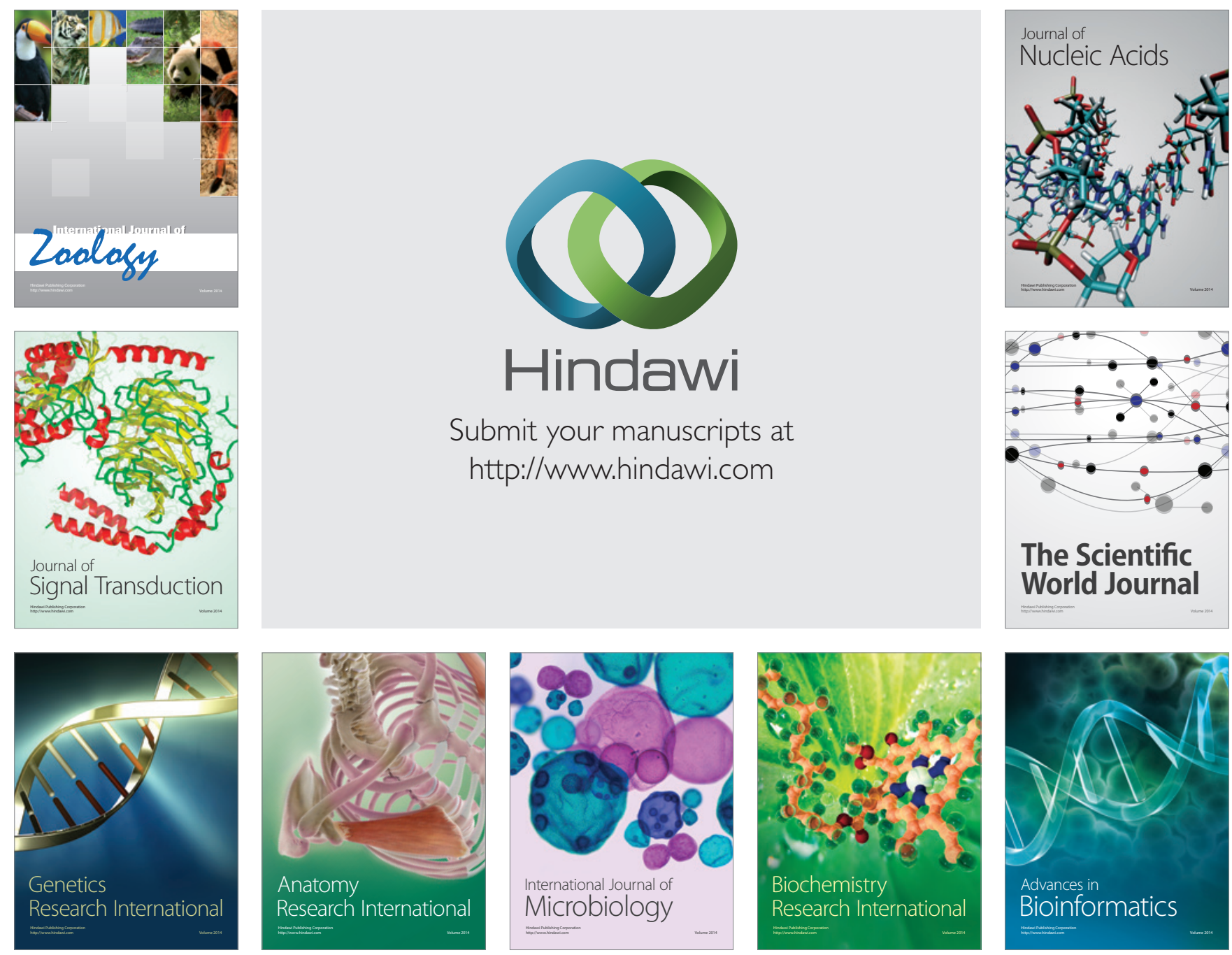

The Scientific World Journal
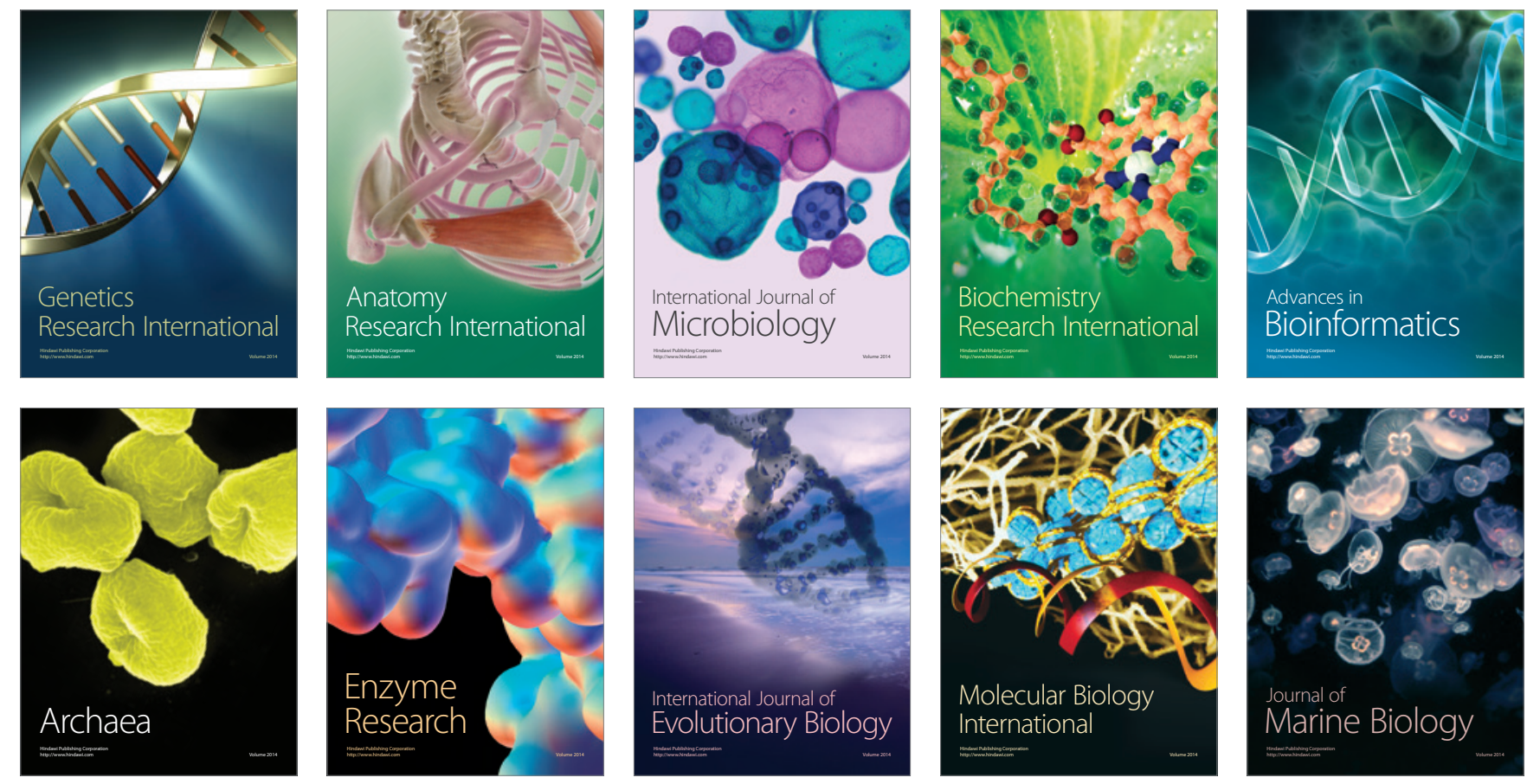